\title{
Corneal transplant outcome in the Palestinian territories: a 2-year follow-up study
}

M Claesson', WJ Armitage ${ }^{2}$, K Olsson-Abdellatif ${ }^{3}$ and N Sargent ${ }^{3}$

\begin{abstract}
Purpose To review the 2-year corneal transplant outcome in a cohort of patients from the Palestinian territories transplanted at the St John Eye Hospital in East Jerusalem in 2001-2002.

Methods Two-year follow-up data were recorded on the Swedish Corneal Transplant Register for 99 of the 161 patients originally transplanted. Multiple regression analyses (linear and logistic) were carried out to determine the influence of diagnosis, preoperative risk factors, and postoperative complications on graft survival and visual outcome.

Results The incidence of preoperative risk factors in keratoconus patients was similar to other diagnoses $(P=0.4)$ but they had fewer postoperative complications (39 vs $77 \%, P<0.001$ ). Graft survival was higher in the keratoconus group (96 vs $49 \%, P<0.001$ ). Visual outcome was also better with $47 \%$ of patients achieving VA $\geqslant 0.5(6 / 12)$ whereas $88 \%$ of the other patients had VA $\leqslant 0.2(6 / 30)$. However, $49 \%$ of these other patients did gain at least one Snellen line. Conclusion Indication had the strongest influence on graft survival and visual outcome. Although the overall results were not so good as in Sweden, the majority of Palestinian patients did show some improvement in vision after corneal transplantation.
\end{abstract}

Eye (2009) 23, 1423-1426; doi:10.1038/eye.2008.263; published online 15 August 2008

Keywords: corneal transplantation; graft survival; visual outcome; penetrating keratoplasty

\section{Introduction}

Penetrating keratoplasty (PK) is a procedure that demands large resources. Apart from the need for tissue, which is often in short supply, PK also requires several hospital appointments, as the healing process and rehabilitation are long. The final result after PK cannot be assessed until at least 2 years after the operation. The outcome after PK is influenced by preoperative risk factors, operating techniques, postoperative complications as well as other factors. ${ }^{1,2}$ There are relatively few follow-up studies in the literature concerning the outcome of ophthalmic care in developing countries $^{3-6}$ and, as a consequence, little is known about the particular conditions affecting results after penetrating keratoplasty and other surgical procedures in those countries. Because of this it is important to establish informed routines for patient selection, postoperative clinical management, and follow-up.

In 2001-2002, one of us (MC) worked as a cornea consultant at the St John Eye Hospital in East Jerusalem. This hospital is the largest single provider of ophthalmic services to the just under four million Palestinians of the Palestinian territories (Gaza, West Bank, and East Jerusalem). Corneal transplantation had been done infrequently in the preceding years, mainly due to a lack of donor corneas. ${ }^{7}$ During 2001-2002, however, a regular supply of about three corneas per week was provided by Tissue Banks International in Baltimore MD. With the help of a fellow from the United States 161 PKs were performed during this time. These operations were included in the Swedish Corneal Transplant Register ${ }^{8}$ to allow comparison with PK carried out in Sweden. We have already described differences between these two populations in the distribution of indications for PK, the severity of disease and preoperative visual acuity. ${ }^{1}$ The purpose of this paper is to report on the 2-year follow-up of the Palestinian patients.
${ }^{1}$ Department of Ophthalmology, Sahlgrenska University Hospital, Mölndal, Sweden

${ }^{2}$ Department of Clinical Science, University of Bristol, Bristol, UK

${ }^{3}$ St John Eye Hospital, Jerusalem, Israel

Correspondence:

M Claesson,

Department of

Ophthalmology,

Sahlgrenska University

Hospital,

S-431 80 Mölndal,

Sweden

Tel + 4631 3431000;

Fax +4631825710.

E-mail: margareta.l.

claesson@vgregion.se

Received: 16 April 2008

Accepted in revised form: 21 July 2008

Published online: 15 August 2008 


\section{Materials and methods}

\section{Patients and data collection}

Of the cohort of 161 patients, ${ }^{1} 62$ were lost to follow-up. Patients with restricted access to Jerusalem were followed up by the St John static and mobile satellite clinics in Gaza and the West Bank.

The forms developed for the Swedish Corneal Transplant Register to report data preoperatively and at 2 years postoperatively were used for data collection. The data were added to the Swedish register, which is now held as a web-based application administered by EyeNet Sweden (www.eyenetsweden.se). Data were then downloaded into a custom designed MS-Access database for subsequent analysis. Data for the 2-year follow-up included patient availability, date of examination, whether the graft was still functioning, postoperative complications (rejection, suture-related problems, glaucoma, infection, recurrence of original disease, cataract surgery, miscellaneous), and best corrected visual acuity in both eyes.

\section{Statistical analysis}

At the time of analysis data were available for 99 of the 161 patients that underwent corneal transplantation during 2001-2002. Not all of these could be included in the analyses owing to missing data, which explains why the numbers of patients in the different analyses varied. Univariate and multiple regression (linear and binary logistic) analyses were carried out using SPSS v14 software. Stepwise procedures were not used for multiple regression and all explanatory variables were entered into the model. Medians are quoted with range, means with standard deviation, regression coefficients $(b)$ with standard errors (se $(b))$ and odds ratios (ORs) with $95 \%$ confidence intervals $(95 \% \mathrm{CI})$. ORs $>1$ signify increased likelihood/risk of an event occurring and $\mathrm{OR}<1$ signify reduced likelihood/risk. The level of significance was set at $5 \%$.

\section{Statement of ethics}

We certify that all applicable institutional and governmental regulations concerning the ethical use of human volunteers were followed during this research.

\section{Results}

\section{Patients}

Patients with keratoconus constituted the single largest group. Because of small numbers in the other groups of indications, it was decided to split the patients into two groups, namely 'keratoconus' and 'other indications'. This latter group comprised bullous keratopathy, regraft, and miscellaneous diagnoses. This was justified because the outcomes for these diagnoses were similar in Swedish patients. ${ }^{8}$ The two groups were now of similar size with 47 (49\%) keratoconus patients and 49 (51\%) 'other indications'. Three patients had no recorded indication. The distribution of indications in the 62 patients lost to follow-up was similar with $46 \%$ keratoconus and 54\% 'other indications' (Fisher's exact test, $P=0.7)$. The median ages of the two follow-up groups were 18 years (range: 10-36 years) for keratoconus and 65 years (range: 5-86 years) for 'other indications'. Age was clearly not independent from indication and was therefore not used as an explanatory variable in the analyses. A single patient with Fuchs' dystrophy was excluded as this indication has a better prognosis than bullous keratopathy or regraft. ${ }^{2,8}$

Preoperative risk factors, including inflammation, vascularization, infection and glaucoma, were grouped together, again owing to small numbers in each category. The proportions of patients with preoperative risk factors were $20 \%$ in the keratoconus group and $13 \%$ for 'other indications' (Fisher's exact test, $P=0.4$ ). The distribution of risk factors was similar in the lost to follow-up group (Fisher's exact test, $P=0.3$ ). There was a preponderance of postoperative complications in followed-up patients in the 'other indications' group, where $77 \%$ of patients had some form of complication, compared with only $39 \%$ of patients in the keratoconus group (Fisher's exact test, $P<0.001)$.

\section{Graft survival}

Indication had a large influence on graft survival with $96 \%$ functioning grafts in the keratoconus group and only $49 \%$ for the 'other indications', which is reflected in a high OR in the logistic regression analysis (OR, 65.1, 95\% CI: 6.7-635.1, $P<0.001)$. Preoperative risk factors markedly decreased the likelihood of graft survival (OR, $0.07,95 \%$ CI: $0.008-0.63, P=0.02$ ), whereas we could not find any negative influence of postoperative complications (OR, 1.23, 95\% CI: $0.34-4.46, P=0.8$ ).

\section{Visual acuity}

Before PK all 161 patients had visual acuities $\leqslant 0.1$. $(\leqslant 6 / 60)$ in the eye to be operated. After PK, $88 \%$ of 'other indications' patients still had low VA $\leqslant 0.2(\leqslant 6 /$ 30 ), compared with $40 \%$ of keratoconus patients (Figure 1); however, $47 \%$ of keratoconus patients achieved a VA $\geqslant 0.5(\geqslant 6 / 12)$. The chance of achieving VA $>0.2$ was higher in the keratoconus group (OR, 8.4, 95\% CI: 2.6-26.9. $P<0.001)$ but was reduced by 
postoperative complications (OR, 0.35, 95\% CI: 0.12-0.98, $P=0.046)$. The presence of preoperative risk factors did not influence the visual outcome $(\mathrm{OR}, 0.59,95 \% \mathrm{CI}$ : 0.15-2.4, $P=0.5)$.

Figure 2 shows the visual outcome in the individual patients expressed as the lines of change on the Snellen chart. A multiple linear regression model (adjusted $R^{2}$ $0.29)$ showed that the keratoconus patients had a greater gain in Snellen lines $(b=2.86, \operatorname{se}(b)=0.64, P<0.001)$. A negative impact of postoperative complications could not be ruled out $(b=-1.26$, se $(b)=0.65, P=0.056)$. Our study failed to show an influence of preoperative risk factors on change in VA $(b=-1.04$, $\operatorname{se}(b)=0.79, P=0.2)$.

The VA in the operated eye after PK was the same or better than the fellow eye for $74 \%$ of the keratoconus group and $62 \%$ of the 'other indications' group. The presence of postoperative complications reduced the likelihood of achieving this (OR, 0.3, 95\% CI: 0.09-0.94, $P=0.04)$; but neither indication (OR, $1.08,95 \% \mathrm{CI}$ : $0.4-2.9, P=0.9)$ nor preoperative risk factors (OR, 0.97, 95\% CI: $0.26-3.64, P=0.96)$ had any impact on whether

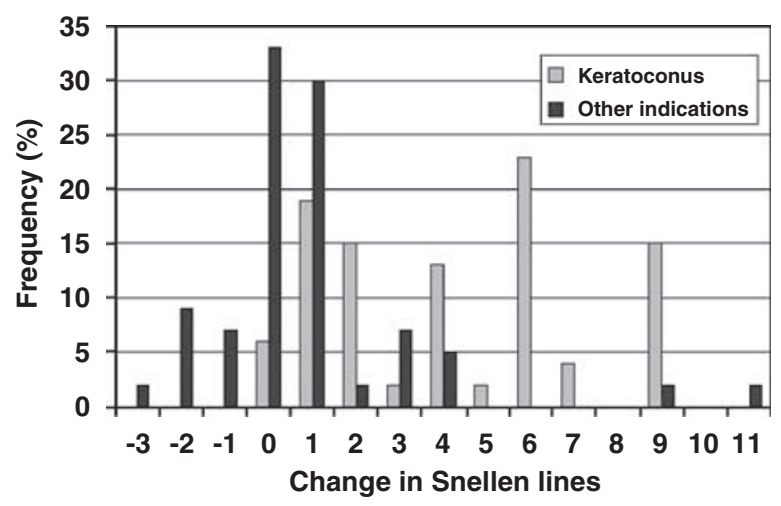

Figure 2 Change in Snellen lines after corneal transplantation for keratoconus and for other indications (excluding Fuchs' dystrophy).

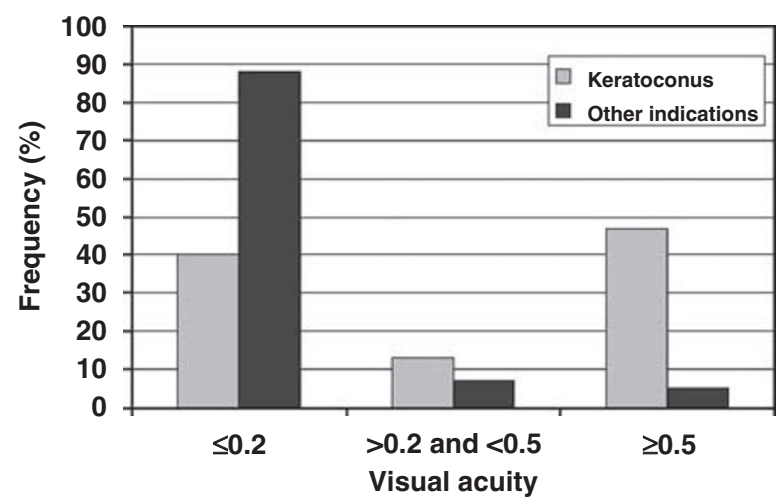

Figure 1 Distribution of postoperative visual acuities for keratoconus and for other indications (excluding Fuchs' dystrophy). the VA in the operated eye was the same or better than the fellow eye.

\section{Discussion}

It is important to monitor the outcome of corneal transplants in areas of poor socioeconomic conditions such as the Palestinian territories as a way of improving and adapting the cornea service according to the specific conditions. Unfortunately, as was initially suspected, follow-up was more difficult in East Jerusalem than in Sweden, partly because of refused access permits to Jerusalem and restrictions on internal movement within the Palestinian territories; but it was possible to followup 99 of the 161 transplanted patients. Keratoconus was the predominant indication for corneal transplantation with $51 \%$ of grafts compared with only $27 \%$ in Sweden. ${ }^{1}$ This is very different from reports from India and Singapore, where keratoconus accounted for only 6.8 and 9.7\% of grafts, respectively. ${ }^{9,10}$ The Palestinian patients with keratoconus were younger than in Sweden, and there was a preponderance of females. ${ }^{1}$ The distributions of diagnoses, age, and gender in the patients available for follow-up were similar to the original cohort. Because of small numbers of patients with indications other than keratoconus, it was decided to group together those transplanted for bullous keratopathy, miscellaneous indications, and regrafts as previous studies had shown that these all have similar outcomes in terms of graft survival and vision. ${ }^{8}$ As a consequence, the single patient with Fuchs' dystrophy was excluded.

The results in the two study groups were very different with the keratoconus group having much better graft survival and visual outcome than the 'other indications' group, albeit not so good as in Sweden. The keratoconus patients had rather more preoperative risk factors, such as glaucoma, vascularization, and inflammation, than would be expected in Sweden. This may be explained by Palestinian patients presenting at hospital much later after the onset of disease owing to the travel restrictions, the cost of transport, delayed referral by doctors, poor patient education, suboptimal public awareness of treatment options, over $50 \%$ poverty and inability to pay for treatment. As a consequence they have far more advanced disease with very poor visual acuity at the first appointment. ${ }^{1}$ Also, the prevalence of vernal catarrh is high in this region, which often causes severe inflammation and vascularization. ${ }^{7}$ Preoperatively a large number of eyes in the 'other indications' group with scars after severe keratitis or trauma had severe ocular surface disease, which substantially increases the risk of graft failure. ${ }^{7,11}$ Studies from Asian countries also show a high proportion of corneal scars and leucomas among the patients undergoing keratoplasty.,10 
However, this disease profile may not truly reflect the overall severity of corneal disease in the Palestinian population as there will be an unknown number of patients that are operated on in Jordan and Egypt who are likely to be more wealthy, better educated, more motivated, and as a consequence have less advanced disease.

Although the diagnosis was the predominant factor influencing both graft survival and postoperative visual acuity, preoperative risk factors mainly had a negative effect on graft survival, whereas postoperative complications led to poorer visual outcome. The keratoconus patients had far fewer postoperative complications than the other indications group (39 vs $77 \%, P<0.001$ ), which is consistent with the results in Sweden and elsewhere. ${ }^{2,8}$ However, we believe that one postoperative complication, rejection, which is an important risk factor for graft failure, ${ }^{12-14}$ was likely to be underreported. This could be because patients with complications were delayed coming to the hospital where they then presented with decompensated corneas, masking the typical signs of rejection. ${ }^{15}$

The better visual outcome in the keratoconus patients was in part related to the absence of age-related retinal disease; but visual improvement in both groups was not as encouraging as would be expected in Sweden. The different corneal storage methods (Optisol-GS in Jerusalem vs organ culture in Sweden) and distances of transportation were unlikely to have influenced visual outcome. ${ }^{16,17}$ It should be noted, however, that the Palestinian patients started with poorer VA: of the patients available for follow-up, $94 \%$ in the keratoconus group and $97 \%$ of patients with other indications had a preoperative VA of counting fingers or worse. There were also problems with postoperative visual rehabilitation with contact lenses and little was done to treat postoperative astigmatism. Despite this, most of them improved after corneal transplantation as expressed by a gain in Snellen lines.

In summary, careful evaluation of the indication before deciding on PK is especially important in patients with many risk factors and where follow-up is difficult. The ideal situation for PK is in an eye that is quiet, with no ongoing inflammation, no vessel activity and no, or well controlled, glaucoma and where thorough follow-up of the patient and treatment regime is possible. If this is not achievable perhaps more conservative medical or surgical treatment should be recommended. However, in spite of the high frequency of risk factors and complications in this cohort of Palestinian patients, the results were in many cases beneficial for individuals.

\section{Acknowledgements}

We thank Tissue Banks International for the supply of corneas and the staff at the St John Eye Hospital and in the outreach clinics for their help and support. This study was funded by institutional support.

\section{Disclosure/Conflict of interest}

None.

\section{References}

1 Claesson M, Armitage WJ. Corneal grafts at St John Eye Hospital, Jerusalem, January 2001-November 2002. Br J Ophthalmol 2004; 88: 858-860.

2 Williams KA, Lowe MT, Bartlett CM, Kelly L, Coster DJ. The Australian Corneal Graft Registry 2007 Report. Flinders University Press: Adelaide, 2007.

3 Yorston DB, Wood ML, Gilbert C. Retinal detachment in East Africa. Ophthalmology 2002; 109: 2279-2283.

4 Tabin GC, Gurung R, Paudyal G, Reddy HS, Hobbs CL, Wiedman MS et al. Penetrating keratoplasty in Nepal. Cornea 2004; 23: 589-596.

5 Tilahun Y, Bejiga A, Alemayehu W. Outcome of corneal transplantation. Ethiop Med J 2005; 43: 27-30.

6 Vanathi M, Sharma N, Sinha R, Tandon R, Titiyal JS, Vajpayee RB. Indications and outcome of repeat penetrating keratoplasty in India. BMC Ophthalmol 2005; 5: 26.

7 De Cock R. Penetrating keratoplasty in the West Bank and Gaza. Eye 1994; 8: 29-34.

8 Claesson M, Armitage WJ, Fagerholm P, Stenevi U. Visual outcome in corneal grafts: a preliminary analysis of the Swedish Corneal Transplant Register. Br J Ophthalmol 2002; 86: $174-180$.

9 Dandona L, Naduvilath TJ, Janarthanan M, Ragu K, Rao GN. Survival analysis and visual outcome in a large series of corneal transplants in India. Br J Ophthalmol 1997; 81: 726-731.

10 Tan DTH, Janardhanan P, Zhou H, Chan Y-H, Htoon HM, Ang LPK et al. Penetrating keratoplasty in Asian eyes: the Singapore Corneal Transplant Study. Ophthalmology 2008; 115: 975-982. e971.

11 Daniels JT, Dart JK, Tuft SJ, Khaw PT. Corneal stem cells in review. Wound Repair Regen 2001; 9: 483-494.

12 Price Jr FW, Whitson WE, Johns S, Gonzales JS. Risk factors for corneal graft failure. J Refract Surg 1996; 12: 134-143; discussion 143-137.

13 Yamagami S, Suzuki K, Tsuru T. Risk factors for graft failure in penetrating keratoplasty. Acta Ophthalmol Scand 1996; 74: 584-588.

14 Inoue $\mathrm{K}$, Amano S, Oshika T, Tsuru T. Risk factors for corneal graft failure and rejection in penetrating keratoplasty. Acta Ophthalmol Scand 2001; 79: 251-255.

15 Alldredge OC, Krachmer JH. Clinical types of corneal transplant rejection. Their manifestations, frequency, preoperative correlates, and treatment. Arch Ophthalmol 1981; 99: 599-604.

16 Frueh BE, Bohnke M. Prospective, randomized clinical evaluation of Optisol vs organ culture corneal storage media. Arch Ophthalmol 2000; 118: 757-760.

17 Varssano D, Russ V, Linhart Y, Lazar M. Air transportation of corneal tissue: experience with local compared to transatlantic donor corneas. Cornea 2005; 24: 674-677. 